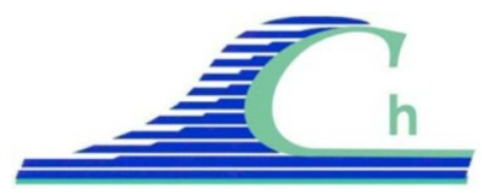

XII ${ }^{\text {ìmes }}$ Journées Nationales Génie Côtier - Génie Civil

Cherbourg, 12-14 juin 2012

DOI:10.5150/jngcgc.2012.060-B @ Editions Paralia CFL

disponible en ligne - http://www.paralia.fr - available online

\title{
Observation vidéo de la dynamique des méga-croissants de plage : plages d'Ánglet (Pays Basque)
}

\section{Florent BIRRIEN ${ }^{1}$, Bruno CASTELLE ${ }^{1}$, Damien DAILLOUX ${ }^{2}$, Vincent MARIEU ${ }^{1}$, Didier RIHOUEY ${ }^{2}$, Timothy PRICE ${ }^{3}$}

\section{UMR CNRS 5805 EPOC, Université Bordeaux 1,} Avenue des Facultés, 33405 Talence Cedex, France.

f.birrien@epoc.u-bordeaux1.fr

2. CASAGEC INGENIERIE,

4 Route de Pitoys, Les pyramides - ZA de Maignon, 64600 Anglet, France.

3. UTRECHT University,Faculty of Geosciences,

P.O. box 80115, 3508 TC Utrecht, The Netherlands.

\section{Résumé :}

Les méga-croissants de plage (megacusps) sont couramment observés le long des hauts de plages sableuses assez pentues. Ils sont la signature érosive des courants d'arrachement au niveau du trait de côte. Dans ce papier nous présentons 8 mois de suivi par imagerie vidéo des comportements des systèmes barre subtidale/megacusp le long les plages Nord d'Anglet. Au Nord de la zone d'étude, dans la région la plus dynamique et fortement contrainte par une digue, ces systèmes sont régulièrement couplés en phase, inférant que la dynamique des megacusps est en grande partie contrôlée par la barre sous-marine.

\section{Mots-clés :}

Méga-croissants de plage - Morphodynamique - Imagerie vidéo - Barres en croissant Trait de côte - Plage sableuse - Erosion - Couplage

\section{Abstract :}

Megacusps are ubiquitous patterns in the sand along steep beach faces. They are erosive signature at the shoreline of the presence of rip currents. In this paper we present an 8months video monitoring of both the nearshore sandbars and the megacusps at the Northern beaches of Anglet. In the northern region, where the most important beach changes were observed as a results of the coastal structures constrains, megacusps were found, most of the time, to couple in phase with the nearshore crescentic sandbars, suggesting that megacusp dynamics is essentially controlled by the evolving nearshore bathymetry.

\section{Keywords :}

Megacusps - Morphodynamics - Video imagery - Crescentic bars - Shoreline - Sandy beaches - Erosion - Coupling 


\section{Introduction}

Les méga-croissants de plage (par la suite appelés megacusps) sont observés en haut d'estran le long des littoraux sableux ayant une pente de haut de plage importante. Ces structures quasi-périodiques ont typiquement une longueur d'onde de $\mathrm{O}(100 \mathrm{~m})$ et une amplitude de $\mathrm{O}(10 \mathrm{~m})$ dans l'axe perpendiculaire à la côte. Ce sont des oscillations du trait de côte, en forme de croissant, souvent couplées à la morphologie subtidale (SHORT, 1999). Ces structures sont importantes car elles impliquent souvent des pics d'érosion locale du haut de plage et parfois du cordon dunaire lors d'épisodes de fortes tempêtes (THORNTON et al., 2007). Ces pics d'érosion sont couramment appelés hotspots dans la littérature. Malgré l'importance de ces structures d'un point de vue scientifique et d'un point de vue pratique dans le cadre d'aménagements du domaine littoral, la dynamique de ces structures est encore très mal comprise et peu documentée.
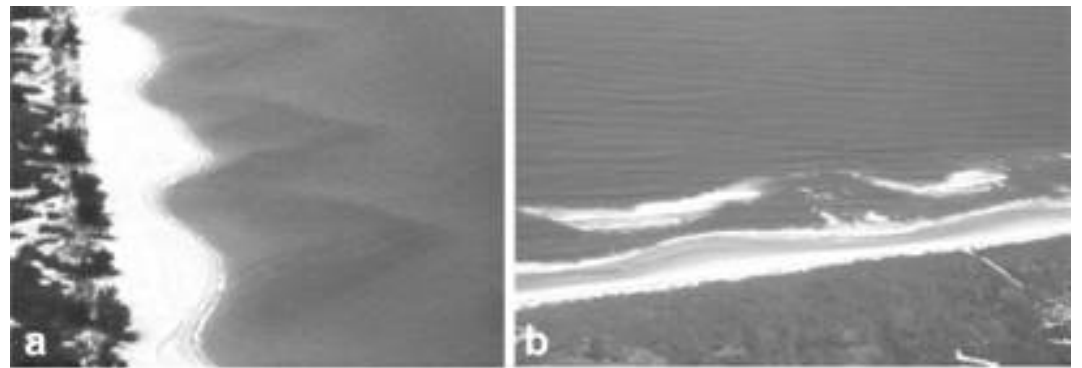

Figure 1. Megacusps couplées (a) en opposition de phase et (b) en phase avec un système de barre en croissant (Photo Prof. A.D. Short dans CASTELLE et al., 2010).

Les observations montrent que les megacusps sont le plus souvent observées en phase avec les chenaux de vidange (figure 1b, voir aussi THORNTON et al., 2007). C'est le cas classique où la megacusp correspond à la signature érosive au niveau du trait de côte de la présence d'un courant d'arrachement guidé par le chenal de vidange. Plus rarement, les megacusps ont été observées en opposition de phase (figure 1a). Les études visant à appréhender leur évolution sont très rares (ORZECH et al., 2011). Toutefois on soupçonne que les mécanismes qui contrôlent ces couplages sont similaires à ceux qui contrôlent les couplages morphologiques en phase et opposition de phase dans les systèmes à double barre (e.g., CASTELLE et al., 2010). Enfin, la formation de megacusps en phase avec les systèmes barre/chenal a récemment été simulée avec succès (CASTELLE \& RUESSINK, 2011), suggérant que les cellules associées aux courants d'arrachement contrôlent leur évolution. La dynamique des megacusps associée à des mécanismes complexes d'auto-organisation reste toutefois mal comprise, notamment à cause du manque de données dépeignant leur évolution.

De par leurs spécificités hydro-sédimentaires et les nombreux aménagements côtiers, les plages Nord d'Anglet présentent la plupart du temps des megacusps bien développées, dont la dynamique n’a encore jamais été étudiée. Ce papier décrit à travers l'imagerie 


\section{XII ${ }^{\text {èmes }}$ Journées Nationales Génie Côtier - Génie Civil \\ Cherbourg, 12-14 juin 2012}

vidéo la dynamique des megacusps le long des plages du littoral Nord Angloy sur une période de 8 mois. Leur évolution est quantifiée et les liens avec la dynamique des barres sous-marines sont discutés.

\section{Suivi vidéo des plages Nord d'Anglet}

Le domaine littoral d'Anglet est une région qui s'étend sur environ 4,5 km de côte fortement anthropisée. La morphologie de ces plages montre l'omniprésence de barres sableuses sous-marines tridimensionnelles, caractéristiques des plages dites intermédiaires dans la classification de WRIGHT \& SHORT (1984). L'environnement est mésotidal (RIHOUEY, 2004) avec des pentes de haut de plage localement fortes ( 1/10), propices au développement de megacusps. La côte Angloye est orientée OuestNord-Ouest (environ $+30^{\circ}$ par rapport l'axe Nord Sud) et est exposée à des houles quasi-normales à la côte avec une moyenne de hauteur significative de $1.57 \mathrm{~m}$ et de période de 7.5 s (ABADIE et al., 2005). Ces houles océaniques ont une forte variabilité saisonnière.

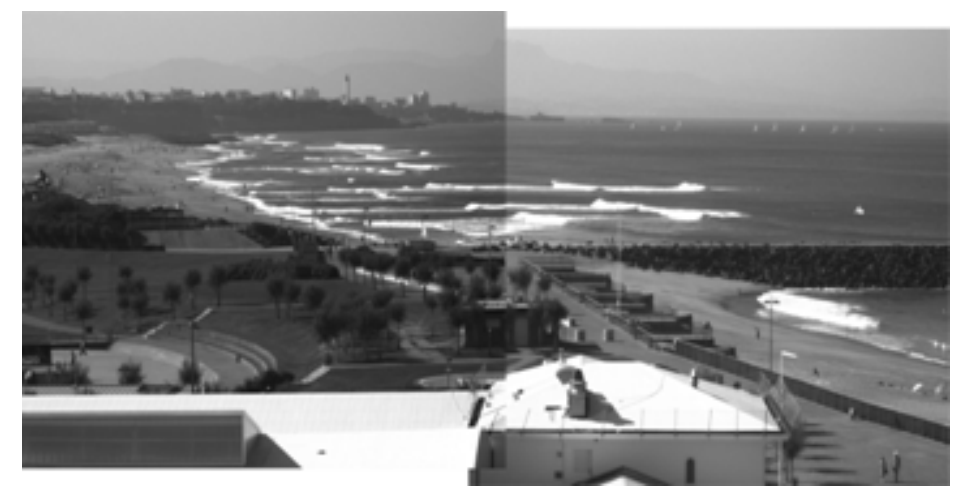

Figure 2. Suivi vidéo des plages Nord d'Anglet (image ‘snapshot’ du 01/10/2011).

Depuis mars 2011, le secteur du nord des plages d'Anglet est équipé d'une station vidéo CASAGEC positionnée sur le phare à l'embouchure de l'Adour. Elle comprend un cluster de 5 caméras dont 4 d'entre elles assurent le suivi journalier de l'évolution d'une zone littorale d'environ 2,5 kilomètres, s'étendant de la plage de la Barre à la plage de l'Océan, avec une emprise de 800 mètres perpendiculairement à la côte (figure 2). Les images vidéo sont successivement redressées et fusionnées pour former une série de timex (pour Time exposure images). Les timex (figure 3) sont des images moyennées (typiquement sur 10 minutes) qui permettent de rendre compte des zones de dissipation d'énergie des vagues et signer la géométrie des barres sous-marines (e.g, VAN ENCKEVORT \& RUESSINK, 2001). Pour notre étude, ces timex nous ont permis de détecter à la fois la présence éventuelle de megacusps par observation des oscillations de la ligne d'eau ainsi que les barres immergées associées. 
La ligne d'eau, définie comme la limite eau-sable, est estimée en considérant les variations du rapport des bandes rouges et bleues du signal lumineux (ALMAR et al., sous presse). Cette méthode s'avère peu coûteuse pour un suivi annuel de trait de côte et surtout appropriée aux caractéristiques des plages d'Anglet. Cela permet de s'affranchir de l'utilisation de méthodes plus coûteuses en temps de calcul pour les géométries de forme plus complexes (e.g., DAILLOUX, 2008). En effet, les plages d'Anglet sont caractérisées par un sable fortement rouge et une pente intertidale suffisamment importante qui permet de s'affranchir de la détection de l'interface 'eau - sable mouillé sable sec’ ou de la présence de bancs de sables émergés. A noter que certains problèmes notamment dus à la qualité de l'image (conditions météorologiques, hydrodynamiques et d'ensoleillement) ou des structures (antennes) ont pu être filtrés excepté ceux inhérents aux zones d'ombres géométriques immédiatement au sud des digues où les données ont été ignorées. L’évolution temporelle du trait de côte peut enfin être réalisée à partir de notre série de timex en fixant un niveau de marée à des fins de comparaison. Une étude préalable basée sur une maximisation de l'amplitude (déviation standard) des oscillations de la ligne d'eau relatif à un marnage donné a permis de retenir le niveau de mi-marée pour observer au mieux les oscillations du trait de côte. Ce niveau universel pour les cycles de mortes-eaux et vives-eaux permet un suivi quotidien du trait de côte. Cependant le risque de travailler à niveau de marée fixe ne nous permet pas d'envisager les fluctuations de l'élévation du niveau d'eau moyen dues aux conditions hydrodynamiques et climatiques, c'est pourquoi (sauf indiqué) nous raisonnerons en termes de position relative moyenne pour le trait de côte.

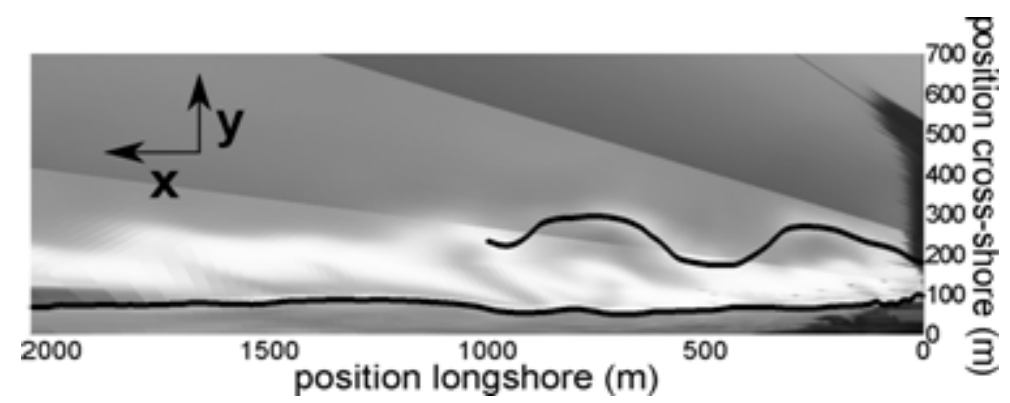

Figure 3. Timex du 23/06/2011 à 16 h avec détection de trait de côte et barre immergée partielle. Le repère est centré sur la digue au Nord de la plage.

Dans cette étude, la géométrie des barres sous-marines, déduites des zones de dissipation par déferlement, a pu être détectée grâce à l'algorithme semi-automatique BLIM (PAPE, 2008) appliqué aux timex redressés et pré-filtrés (figure 3). Cette méthode a été appliquée avec succès à différentes études d'évolutions de barres sableuses (e.g., PRICE \& RUESSINK, 2011). Les zones de dissipation fluctuent avec les conditions hydrodynamiques, le déferlement sur la barre sous-marine n'étant observé que pour des conditions de vagues suffisamment énergétiques. 

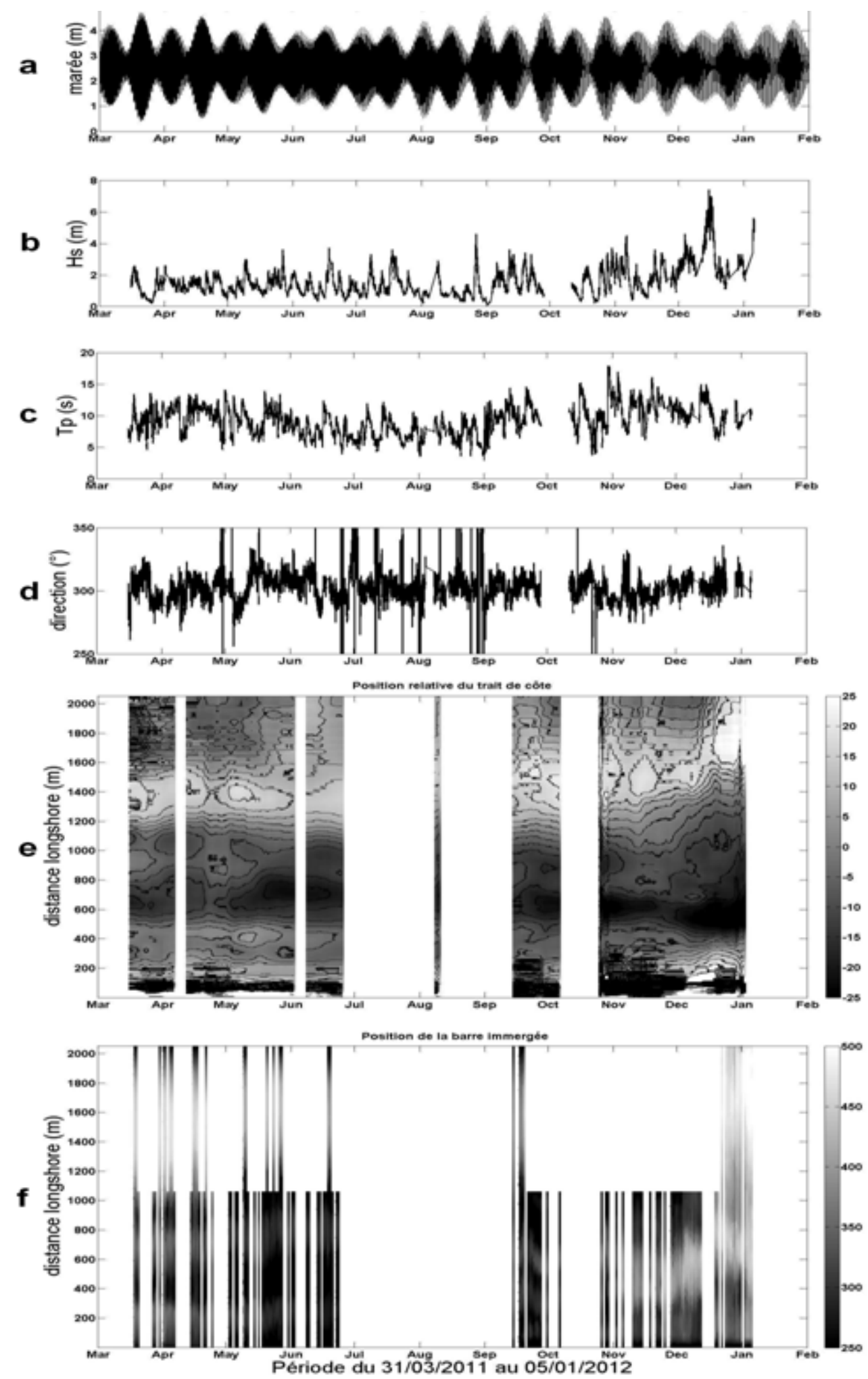

Figure 4. Séries temporelles de marée isuue du modèle Ttide (a), des paramètres hydrodynamiques (Hs (b), Tp (c) et direction (d), issus de la bouée $n^{\circ}$ 06402), du trait de côte (e) et de la barre immergée ( $f$ ) (conformément au repère établi figure 3 ). 


\section{Résultats}

La figure 4 présente les séries temporelles d'évolution du trait de côte (e) et de la barre immergée (f) sur la portion de plage étudiée (figure 3) en relation avec les conditions hydrodynamiques (b, c, d) et du marnage (a) pour la période du 16/03/2011 au 05/01/2012. Pendant cette période, les houles étaient en moyenne normales à la côte (302 $)$, avec une hauteur significative (Hs) de 1,52 m et une période (Tp) de $9.1 \mathrm{~s}$ et sensiblement moins énergétiques ( $\mathrm{Hs}=1.25 \mathrm{~m}, \mathrm{Tp}=8.56 \mathrm{~s}$ ) pour la période estivale (avril à octobre). Les discontinuités observées dans les données sont exclusivement dues aux arrêts épisodiques de caméra en ce qui concerne le trait de côte. La détection de la barre sous-marine est plus délicate et n’est possible qu'en cas de déferlement en présence de houles consistantes.

Les conditions de vagues relativement peu énergétiques sur l'année nous permettent de conclure sur une certaine stabilité significative des systèmes de megacusps et de barres sous-marines. La morphologie de plage a toutefois connu un bouleversement important au passage d'un épisode de tempête en fin de période de suivi en décembre, associé à des houles supérieures à $6 \mathrm{~m}$, provoquant une érosion du trait de côte et une migration de la barre externe vers le large. Le trait de côte présente globalement 2 voire 3 oscillations dont une majeure centrée initialement en $\mathrm{x}=700 \mathrm{~m}$ qui resta stable jusqu'à l'été puis qui migra progressivement depuis octobre vers une position à $\mathrm{x}=500 \mathrm{~m}$ en décembre. Concernant la barre immergée, 2 longueurs d'onde de croissant étaient bien visibles sur la zone Nord ( $x<1000 \mathrm{~m}$ ) en début de période (figure $4 \mathrm{f})$, l'une proche de la digue centré en $\mathrm{x}=200 \mathrm{~m}$ et l'autre centré en $\mathrm{x}=700 \mathrm{~m}$. On remarque que deux megacusps sont aussi couplées en phase avec ces deux systèmes en début de période (figure 4e). Ces systèmes ont ensuite migré progressivement vers le sud puis vers le Nord avant que les deux systèmes s'apparient vers la fin de l'été. Même si ces observations ne permettent pas d'expliquer pourquoi nous observons l'appariement à la fois des systèmes de megacusps et de barres, nous pouvons soupçonner que les systèmes de barre sous-marine tentent en permanence de s'auto-organiser en des longueurs d'onde régulières (le système le plus proche de la digue ayant initialement des dimensions trop faibles). Les megacusps dans cette zone de notre site d'étude se sont alors naturellement adaptées aux changements de la morphologie subtidale. La partie Sud de la zone d'étude est plus stable. Elle est délimitée par une barre externe immergée en croissant relativement profonde, localisée en moyenne en y=500 m et de longueur d'onde de $1000 \mathrm{~m}$. Cette barre, qui n'est visible que pendant les houles significatives sur la zone (figure 4f), n’a pas beaucoup évolué sur l'année sauf pendant la tempête de mi-décembre. La barre interne présente des systèmes barre/chenal plus mobiles (non détectés dans cette étude), avec les chenaux de vidange majoritairement transverses (orientés Sud) et assez peu développés. Ainsi, la signature érosive au niveau du trait de côté est peu prononcée (absence de megacusps bien développées). 

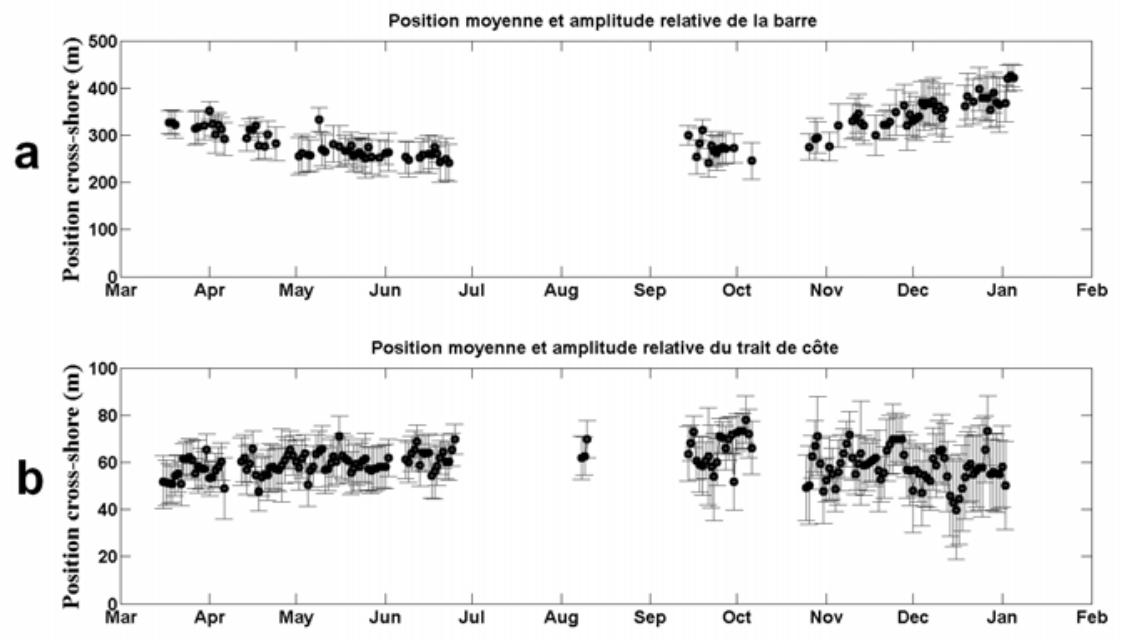

Figure 5. Position moyenne de la barre immergée (a) et du trait de côte (b) et leurs déviations standards respectives pour les 1000 mètres au Nord de la plage.

La figure 5 montre l'évolution temporelle de la position du trait de côte et de la barre sous-marine en termes de position moyenne transversale et de déviation standard de sa position (i.e., proxy de sa tridimensionnalité). Pendant la période estivale, la barre a tendance à migrer vers la plage tandis qu'on observe une accrétion de la plage. A l'arrivée des houles consistantes à partir du mois d'octobre, la barre en croissant migre progressivement vers le large tandis que la plage s'érode, le sédiment érodé étant transporté vers la plage sous-marine. Il est difficile de conclure en termes de déviation standard des oscillations (amplitudes relatives). On observe cependant une faible tendance des systèmes à se tridimensionnaliser sous l'effet de la houle, mis à part à proximité de la digue qui contraint la fixation de courant d'arrachement (et donc d'un chenal) le long de celle-ci (CASTELLE \& COCO, sous presse).

\section{Conclusion}

Dans cette étude l'imagerie vidéo nous a permis, via une série de timex, de décrire les comportements des systèmes de megacusps et de la barre subtidale en croissant sur le domaine littoral Nord Angloy. La zone concernée peut se décomposer en 2 zones caractéristiques. L'une au sud ( $x>1000 \mathrm{~m})$ très stable sur l'année, présentant une barre sableuse externe profonde et de grande longueur d'onde, associée à une barre interne présentant des systèmes barre/chenal plus courts et plus mobiles qui ont peu d'influence sur la dynamique des megacusps. Cette dynamique contraste avec celle plus au nord $(\mathrm{x}<1000 \mathrm{~m})$ où la dynamique des megacusps semble être essentiellement contrôlée par la barre sous-marine. On y observe des couplages classiques en phase entre la barre et les megacusps. Il est important de noter que notre période d'étude était assez courte et caractérisée par les conditions assez peu énergétiques. Il sera intéressant de poursuivre 
ce suivi afin de dépeindre en détail la dynamique de megacusps et ses variabilités saisonnière et interannuelle.

\section{Références bibliographiques}

ABADIE S., BUTEL R., DUPUIS H., BRIERE C. (2005), Paramètres statistiques de la houle au large de la côte sud-aquitaine. C.R. Geoscience, 337, pp 769-776. doi:10.1016/j.crte.2005.03.012

ALMAR R., RANASINGHE R., SENECHAL N., BONNETON P., ROELVINK D., BRYAN K., MARIEU V., PARISOT J-P. (sous presse), Video based detection of shorelines at complex meso-macro tidal beaches. Journal of Coastal Research. CASTELLE B., COCO G. (sous presse) The morphodynamics of rip channels on embayed beaches. Continental Shelf Research.

CASTELLE B., RUESSINK B.G. (2011), Modeling formation and subsequent nonlinear evolution of rip channels: time-varying versus time invariant wave forcing. Journal of Geophysical Research - Earth Surface, 116, F040048, doi:10.1029/2011JF001997.

CASTELLE B., RUESSINK B.G., BONNETON P., MARIEU V., BRUNEAU N., PRICE T.D. (2010), Coupling mechanisms in double sandbar systems, Part 1: Physical explanation and coupling patterns. Earth Surface Processes and Landforms, 35(7), pp 771-781.

DAILLOUX D. (2008), Video measurements of the Adour plume dynamic and its surface water optical characteristics. Thèse, Université de Pau et des Pays de l'Adour.

ORZECH M.D, RENIERS A.J.H.M., THORNTON E.B., MACMAHAN J. (2011), Megacusps on rip channel bathymetry: Observations and modeling. Coastal Engineering, n58, pp 890-907. doi:10.1016/j.coastaleng.2011.05.001

PAPE L. (2008). BLIM Toolbox Manual. Utrecht University, the Netherlands, p 41.

PRICE T.D., RUESSINK B.G. (2011), RUESSINK, State dynamics of a double sandbar system. Continental Shelf Research, 31, pp 659-674. doi:10.1016/j.csr.2010.12.018

RIHOUEY D. (2004), Analyse statistique de l'évolution morphodynamique des plages sableuses. Application aux sites d'étude du Programme National d'Environnement Côtier et aux plages d'Anglet. Thèse, Université de Pau et des Pays de l'Adour.

SHORT A.D. (1999), Handbook of beach and shoreface morphodynamics. John Wiley and Sons, Ltd., NewYork, 379 p.

THORNTON E.B., MACMAHAN J., SALLENGER A.H. JR. (2007), Rip currents, mega-cusps, and eroding dunes. Marine Geology, 240, pp151-167. doi:10.1016/j.margeo.2007.02.018

VAN ENCKEVORT I.M.J., RUESSINK G. (2001), Effect of hydrodynamics and bathymetry on video estimates of nearshore sandbar position. Journal of Geophysical Research, 106 C8, pp 16,969-16,979. doi:10.1029/1999JC000167

WRIGHT L.D., SHORT A.D (1984), Morphodynamics variability of surf zones and beaches: a synthesis. Marine Geology, 70, pp 251-285. doi:10.1016/0025-3227(86)90005-8 


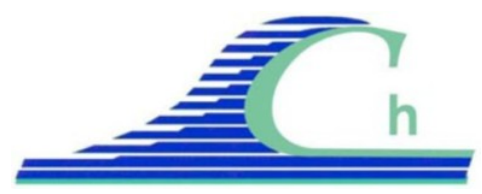

XII ${ }^{\text {èmes }}$ Journées Nationales Génie Côtier - Génie Civil

Cherbourg, 12-14 juin 2012

DOI:10.5150/jngcgc.2012.061-C @ Editions Paralia CFL

disponible en ligne - http://www.paralia.fr - available online

\title{
Mise en ouvre d'un système de numérisation 3D mobile pour le suivi détaillé d'un linéaire de $3.5 \mathrm{~km}$ de falaises à Carry-le-Rouet (Bouches-du-Rhône)
}

\author{
Bertrand CHAZALY ${ }^{1}$
}

\author{
1. Fugro Geoid SAS, 12 rue des Frères Lumière, 34380 Jacou, France. \\ b.chazaly@fugro.com
}

\section{Résumé :}

Développé par le groupe Fugro, Boat-Map ${ }^{\mathrm{TM}}$ est un système couplant un sondeur bathymétrique multifaisceaux à un scanner 3D mobile. C'est un système modulable permettant d'enregistrer et de géoréférencer une image 3D intégrale des surfaces audessus et sous l'eau, fournissant une topographie dense, précise et exhaustive des espaces maritimes et fluviaux.

Mis en œuvre à Carry-le-Rouet fin novembre 2011, le système a produit un semis de points 3D couvrant un linéaire de $3,5 \mathrm{~km}$ de falaises, à une densité moyenne et une précision de $5 \mathrm{~cm}$. Parallèlement, une série de prises de vues photographiques haute résolution a été enregistrée et géo-référencée.

Le traitement des données aboutit à la fourniture de produits cartographiques exploitables : modèles maillés 3D, développé orthophotographique du linéaire de côte, cartographie des écarts au premier état enregistré.

La mesure 3D dense des élévations des falaises offre un instantané détaillé de leur morphologie, de précision centimétrique. La répétition de ces mesures à intervalle régulier permet un suivi de l'évolution de cette morphologie, d'où sont tirées des caractéristiques des mécanismes d'érosion, afin d'établir une typologie des instabilités de falaises et de qualifier l'aléa.

\section{Mots-clés :}

Centrale inertielle - GPS - Scanner 3D - Bathymétrie multifaisceaux Lasergrammétrie - Numérisation 3D - Modélisation numérique - Auscultation - Trait de côte - Falaises

\section{Introduction}

En région Provence-Alpes-Côte d'Azur, une approche des risques naturels, liés aux instabilités de falaises côtières a été jugée nécessaire compte tenu des enjeux (sécurité des personnes et des biens, impact socio-économique, environnement). Les falaises de Carry-le-Rouet font parties des 4 sites représentatifs du littoral à falaise de la région.

Afin d'établir une typologie des instabilités de falaises et de qualifier l'aléa, Le Bureau de Recherches Géologiques et Minières réalise des investigations de terrain, préalables à une compréhension des phénomènes qui doit aboutir à une caractérisation de l'érosion et 